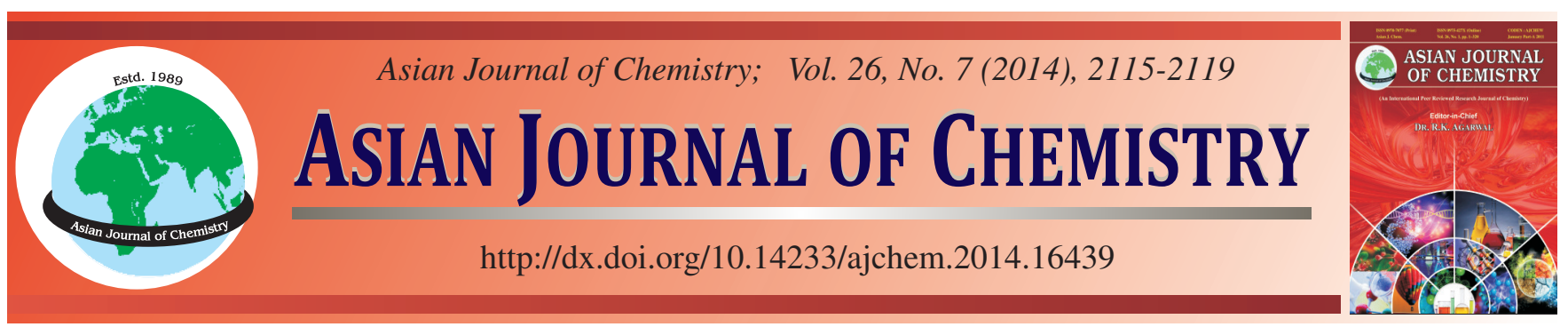

\title{
Preparation and Thermal Properties of Poly(phosphazene-aryl ester) Containing Cyclotriphosphazene Structures
}

\author{
ZhengPing Zhao*, JiAnBing Ji and FengYing Yu
}

Zhijiang College of Zhejiang University of Technology, Hangzhou 310024, P.R. China

*Corresponding author: E-mail: sjzhaolei@163.com

\begin{abstract}
Newly aromatic poly(phosphazene-aryl ester) was prepared by polycondensation reaction of synthesized aromatic diols: 1,1,3,5tetraphenoxy-3,5-bis(4-hydroxyphenoxy) cyclotriphosphazene with terephthaloyl dichloride. The chemical structure of the synthesized diols and polyphenyl esters were characterized by elemental analysis, ${ }^{1} \mathrm{H}$ and ${ }^{31} \mathrm{P}$ NMR and FTIR techniques, whereas their thermal and crystallize properties were determined by DSC, TGA and XRD techniques. Compared to conventional $p$-oxybenzoyl homopolyester, the poly(phosphazene-aryl ester) shows excellent thermal stability and solubility in polar protic solvents. Poly(phosphazene-aryl ester) shows two thermal degradation in the temperature range $150-600{ }^{\circ} \mathrm{C}$ while the monomer, indently its structure, shows the first maximum rate of thermal decomposition temperature around $150-300^{\circ} \mathrm{C}$, which may be due to the decomposition of the P-O-C bond.
\end{abstract}

Keywords: Polyphosphazene, Polyphenyl esters, Preparation, Thermal properties.

\section{INTRODUCTION}

Polyphosphazene as an organic-inorganic hybrid polymer is a class of novel macromolecules containing alternate phosphorus-nitrogen single and double bonds with two changing organic side groups to be prepared new specific functions materials. Because the phosphorus-nitrogen bonds are extremely flexible due to the low torsional energy and a large variety of side groups, polyphosphazenes can be made with a wide range of chemical and physical properties, like excellent heat resistance, cold-resistant, water-tolerant, solvent resistance, radicalization resistance and flame retardant performances in many areas ${ }^{1-7}$.

In our research, we decide to synthesize a new aromatic phosphazene diols monomer and use it for the synthesis of new fully aromatic poly(phosphazene-aryl ester), in order to investigate its thermal behavior. It will be used as the additiontype monomers selected on the basis of the tribopolymerization concept in the further vapor phase lubrication study. Their physical chemistry properties including structures, thermal properties, crystallization behaviorand degradation behavior are studied.

\section{EXPERIMENTAL}

Hexachlorocyclotriphosphazene (HCCP) (synthesized as described in the literature ${ }^{8}$ ) was recrystallized from dry hexane followed by sublimation $\left(60^{\circ} \mathrm{C}, 0.05 \mathrm{~mm} \mathrm{Hg}\right)$ twice before use $\left(\mathrm{mp}=112.5-113^{\circ} \mathrm{C}\right)$. Phenol, tetrahydrofuran $(\mathrm{THF}), 4,4^{\prime}-$ sulfonyldiphenol (BPS), N-methyl-2-pyrrolidinone (NMP) and triethylamine (TEA) were obtained from Sinopharm Chemical reagent Co., Ltd (Shanghai China). Terephthaloyl chloride (TPC) was purified by recrystallization form anhydrous hexane. All chemicals and solvents were provided commercially by Sinopharm Chemical Regents Co. Ltd (China) and used without further purification unless otherwise noted. All glassware was dried in an oven under vacuum before use.

Synthesis of 1,1,3,5-tetraphenoxy-3,5-bis(4-hydroxyphenoxy)cyclotriphosphazene: The preparation of diols monomer involved two steps: In the first step, $\mathrm{NaH}$ (9.60 g, $0.40 \mathrm{~mol}$ ) was dissolved in $20 \mathrm{~mL}$ tetrahydrofuran under stirring and nitrogen atmosphere. To this, a solution of tetrahydrofuran $(50 \mathrm{~mL})$ dissolving phenol $(37.65 \mathrm{~g}, 0.40 \mathrm{~mol})$ was added dropwise. The temperature of reaction mixture was maintained at room temperature for $2 \mathrm{~h}$ and then added this solution dropwise to a solution of hexachlorocyclotriphosphazene $(34.80 \mathrm{~g}, 0.10 \mathrm{~mol})$ in $100 \mathrm{~mL}$ of tetrahydrofuran at room temperature for $24 \mathrm{~h}$. Then, after tetrahydrofuran removed by rotary evaporation, the reaction mixture was to be purified by ethanol and deionized water three times. Finally, the product (bis-chlorinetetraphenoxycyclotriph-osphazene) was dried under vacuum at $60{ }^{\circ} \mathrm{C}$ for $12 \mathrm{~h}$. In the second step, a solution of the product I $(45.25 \mathrm{~g}, 0.05 \mathrm{~mol})$ in $100 \mathrm{~mL}$ of tetrahydrofuran was added dropwise to a solution of 4,4'-sulfonyldiphenol $(25.02 \mathrm{~g}, 0.10 \mathrm{~mol})$ and triethylamine $(10.05 \mathrm{~g}, 0.10 \mathrm{~mol})$ in 
$100 \mathrm{~mL}$ of tetrahydrofuran at room temperature. After completion of the addition in $2 \mathrm{~h}$, the mixture was stirred and refluxed at $40{ }^{\circ} \mathrm{C}$ for $12 \mathrm{~h}$. Then, the reaction mixture was cooled to room temperature. tetrahydrofuran was removed by distillation under reduced pressure. The residue was dissolved in acetone, washed three times with deionized water and dried over anhydrous magnesium sulfate. Finally, the diols monomer (product II) was dried under vacuum at $60^{\circ} \mathrm{C}$.

Synthesis of poly(phosphazene-aryl ester): Fully aromatic poly(phosphazene-aryl ester) (PPOB) was synthesized following a procedure as depicted in Scheme-I. $p$-Oxybenzoyl homopolyester (POB) is also synthesized to study compared with poly(phosphazene-aryl ester).

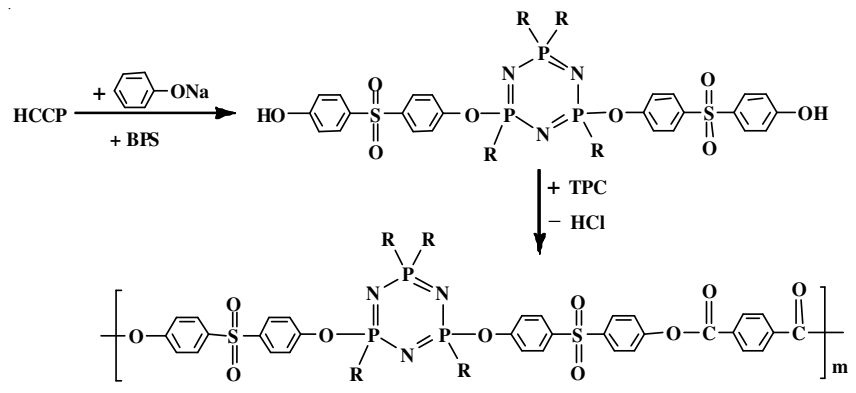

$\mathrm{R}=\longrightarrow \mathrm{O}, \mathrm{m}=1,2,3 \ldots$

Scheme-I: Synthesis of diols monomer and poly(phosphazene-aryl ester)

FT-IR spectra of all the samples were recorded using polymer granule on a Perkin-Elmer Wellesley MA spectrophotometer. The $\left({ }^{1} \mathrm{H}\right.$ and $\left.{ }^{31} \mathrm{P}\right)$ NMR spectra were recorded on a Varian DRX 400 NMR spectrometer with the operating frequency at 400 $\mathrm{MHz}$ using $\mathrm{CDCl}_{3}$ or DMSO as a solvent, using TMS as inner reference and $\mathrm{H}_{3} \mathrm{PO}_{4}(85 \%)$ as external reference. Elemental analysis was carried out using a Heraeus $\mathrm{CHN}-\mathrm{O}$ rapid elemental analyzer with acetanilide as a standard. Thermogravimetric analysis (TGA) was performed on a TGA 7 instrument (Perkin Elmer) thermal analysis system. Sample weight taken was 2-4 mg. DSC analysis was carried out on a Perkin-Elmer Pyris 2 DSC analyzer (Perkin Elmer), at a heating rate of $10^{\circ} \mathrm{C} / \mathrm{min}$ in nitrogen atmosphere. Sample weight taken was $15-20 \mathrm{mg}$. Wide-angle X-ray scattering measurements were performed on a Bruker AXS-D8 Avance X-ray diffractometer with a copper target $(40 \mathrm{kV}, 15 \mathrm{~mA})$. The microstructures of solid residues were recorded using a Cambridge S250MK3 scanning electron microscope (U.K.). The solid residue of the samples degraded to various extents was also prepared using the TGA instrument under nitrogen atmosphere. The materials were heated from room temperature to $600{ }^{\circ} \mathrm{C}$ with a heating rate of $20^{\circ} \mathrm{C} / \mathrm{min}$ and then rapidly cooling the residue to room temperature.

\section{RESULTS AND DISCUSSION}

The fully aromatic polybenzoate reported in Scheme-I were prepared by polycondensation reaction of the diols monomer with the terephthaloyl chloride in the presence of solvent and a base. The diols monomer, which is a new polyphosphazene aromatic diols, was synthesized according to Scheme-I through a two-step route.
The chemical structures of the polyphosphazene-containing monomer synthesized in the study were characterized by elemental analysis, ${ }^{1} \mathrm{H}$ NMR, ${ }^{31} \mathrm{P}$ NMR and FT-IR spectroscopy. The calculated elemental contents of monomer in terms of the formula $\left(\mathrm{C}_{36} \mathrm{H}_{30} \mathrm{~N}_{3} \mathrm{O}_{10} \mathrm{P}_{3} \mathrm{~S}_{2}\right)$ were $\mathrm{C} 52.62 ; \mathrm{H} \mathrm{3.68;} \mathrm{N}$ 15.11; O 19.47; P 11.31; S 7.80 and the analyzed data show that $\mathrm{C}, \mathrm{H}, \mathrm{N}, \mathrm{O}, \mathrm{P}$ and $\mathrm{S}$ are $62.41,3.82,15.69,19.57,10.98$ and 7.33, respectively. It is found that elemental analysis of the synthesized diols monomer is in good agreement with the calculated values. The ${ }^{31}$ P NMR spectrum (Fig. 1) of the diols monomer recorded at room temperature in DMSO, shows two intense peaks $(\delta=7.48 \mathrm{ppm}$, for the two phenol groups linked to the phosphorus atoms; $\delta=19.30 \mathrm{ppm}$, for the phenoxy group and phenylsulfone group linked to the phosphorus atoms). This experimental evidence denotes that the chlorine was completely replaced by two groups. Furthermore, in the ${ }^{1} \mathrm{H}$ NMR spectrum of diols monomer (Fig. 1), the chemical shifts corresponding to the phenolic ring are found at $\delta=6.89$ ppm and $\delta=7.21 \mathrm{ppm} ; \delta=3.04 \mathrm{ppm}$ and $\delta=2.97 \mathrm{ppm}$, as the two doublets prove the presence of the phenoxy groups.

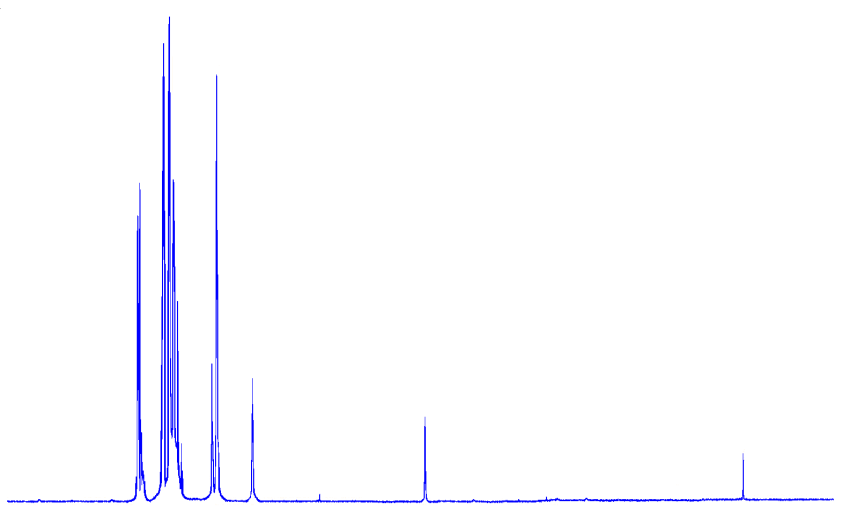

$9.08 .58 .07 .57 .06 .56 .05 .55 .04 .54 .03 .53 .02 .52 .01 .51 .00 .50 .0 \quad \mathrm{ppm}$

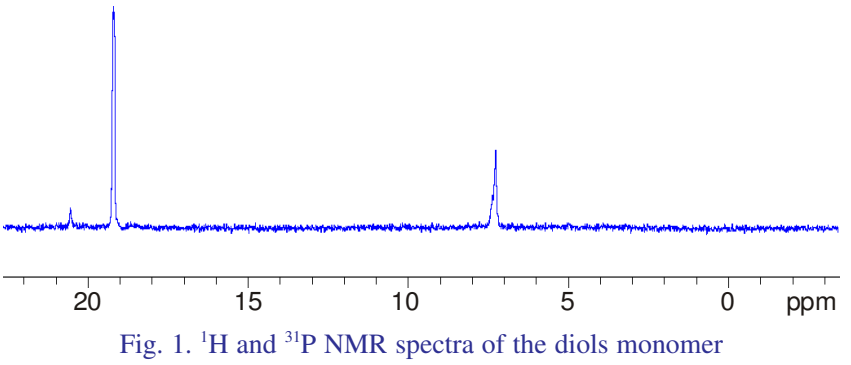

In order to obtain a more complete and reliable chemical characterization, the synthesized monomer, was also investigated by FT-IR technique. The spectrum is shown in Fig. 2. The monomer shows a strong absorption band at $1230 \mathrm{~cm}^{-1}$ due to the $\mathrm{P}=\mathrm{N}$ stretch, while the absorption band at $875 \mathrm{~cm}^{-1}$ due to the $\mathrm{P}-\mathrm{N}$ stretch. It also shows strong absorption peaks at $1150 \mathrm{~cm}^{-1}$, at 940 and $3250 \mathrm{~cm}^{-1}$ due to the $\mathrm{O}=\mathrm{S}=\mathrm{O}$ stretch, $\mathrm{P}-\mathrm{O}-\mathrm{Ar}$ stretch and $\mathrm{O}-\mathrm{H}$ stretching, respectively.

FT-IR spectra of the poly(phosphazene-aryl ester) shown in Fig. 3. present the characteristic absorption bands due to the stretching of the -OH groups along the chain in the range $3500-3000 \mathrm{~cm}^{-1}$, while the stretching of the polyphosphazene groups give a wide band at $1200 \mathrm{~cm}^{-1}$. Typical signals due to the stretching of the $\mathrm{C}=\mathrm{O}$ stretching and $\mathrm{C}-\mathrm{O}$ bending appear 


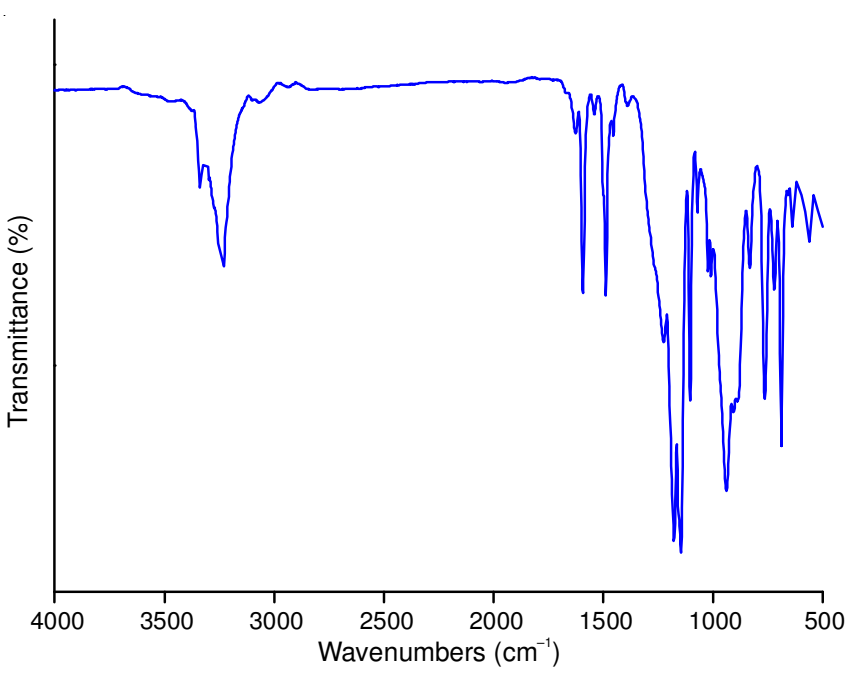

Fig. 2. FT-IR spectrum of diols monomer

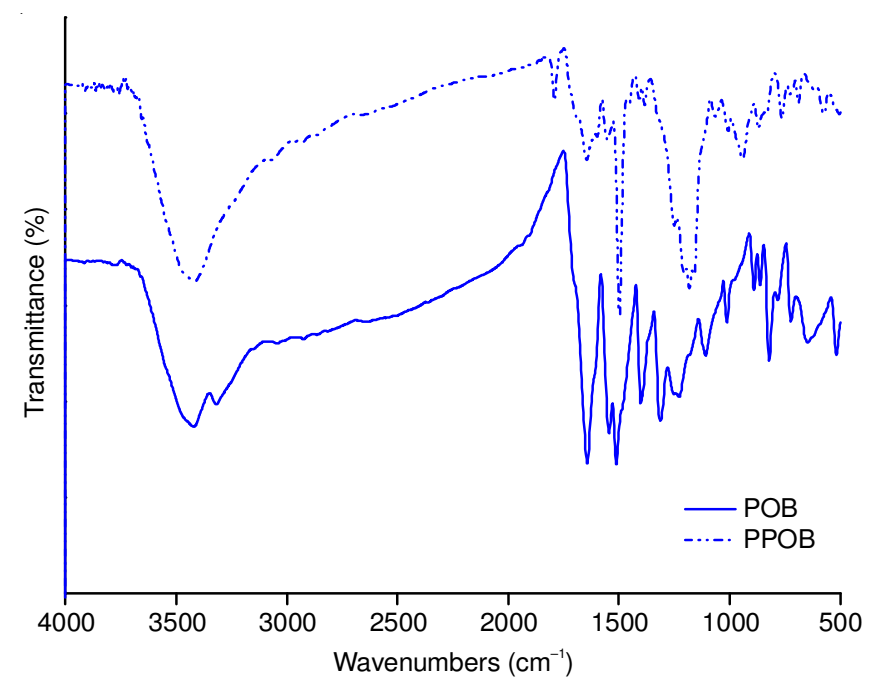

Fig. 3. FT-IR spectrum of the poly(phosphazene-aryl ester)

at 1740 and $1500 \mathrm{~cm}^{-1}$, respectively. Similar FT-IR spectra were achieved for fully aromatic polyphosphazene polyester.

In conclusions, the inspection of elemental analysis, ${ }^{1} \mathrm{H}$ NMR, ${ }^{31} \mathrm{P}$ NMR and FT-IR spectra reveal that the novel poly(phosphazene-aryl ester) synthesized and discussed in the present work can be well characterized and distinguished applying these powerful methods.

Solubility of the poly(phosphazene-aryl ester): The solubility of the fully aromatic poly(phosphazene-aryl ester) was tested in various solvents at $1 \mathrm{wt}$. \% concentration and the results are summarized in Table-1.

TABLE-1

SOLUBILITY BEHAVIOUR ${ }^{1}$ OF THE POLYESTERS IN VARIOUS SOLVENTS ${ }^{2}$

\begin{tabular}{cllllllll}
\hline Solvent & $\mathrm{CHCl}_{3}$ & THF & DMF & DMAc & NMP & DMSO & $\mathrm{H}_{2} \mathrm{SO}_{4}$ \\
\hline POB - & - & - & - & - & - & + \\
PPOB & - & - & \pm & \pm & + & + & + \\
\hline
\end{tabular}

${ }^{1}$ Solubility: (+) soluble at room temperature; $( \pm)$ soluble with worming or swollen; (-) insoluble. ${ }^{2} \mathrm{THF}$ : tetrahydrofuran; DMF: $N, N$-dimethyl formaide; DMAc: N,N-dimethyl acetamide; NMP: N-methyl pyrrolidone; DMSO: dimethyl sulfoxide
The novel poly(phosphazene-aryl ester) was readily soluble in highly polar solvents such as DMF, DMAc, DMSO and N-methyl-2-pyrrolidinone at room temperature or upon heating. The high-solubility nature of it can be explained by the presence of polypolyphosphazene groups. Because of these voluminous groups, the packing of the polymer chains in tight structures through hydrogen bonding between ester groups is prevented and, consequently, the solvent molecules can easily diffuse into the polymer chain. The increased solubility of the poly(phosphazene-aryl ester) is mainly due to their amorphous character.

Crystallinity of poly(phosphazene-aryl ester): In order to study the crystalline or amorphous nature of the polyesters, WAXD measurements at room temperature were performed. Typical wide-angle X-ray diffractograms of the powder polyesters are illustrated in Fig. 4. The curves showed that there are two dispersion peaks at $2 \theta=16.7^{\circ}$ and $23.1^{\circ}$ for aromatic polybenzoate, which indicates it has low crystallinity. But, there are no sharp peaks in the poly(phosphazene-aryl ester). It proves that poly(phosphazene-aryl ester) is amorphous. This can be attributable to the introduction of polyphosphazene groups, which weakens the regular structure of molecular chains, reduces the rigidity and therefore disrupts the crystallization capacity of the polyamides. Thus, the amorphous structure of this polyester also reflected in its excellent solubility.

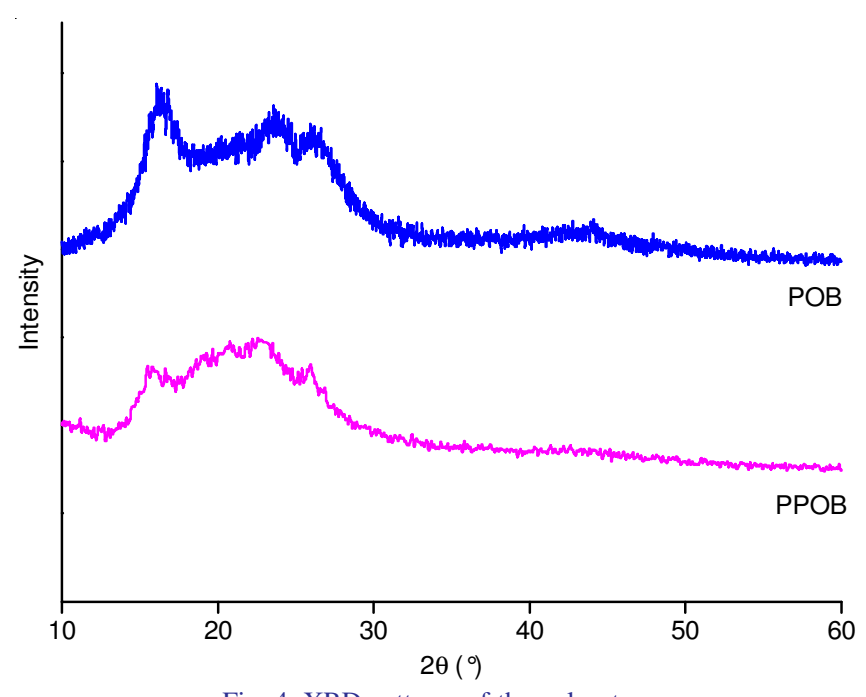

Fig. 4. XRD patterns of the polyesters

Thermal characterization: Thermal properties and stability of the polyesters were studied by DSC and TGA techniques and the data are reported in Table-2. As can be observed, in Table- 2 the poly(phosphazene-aryl ester) is a amorphous sample with a glass transition $\left(\mathrm{T}_{\mathrm{g}}\right)$ of $85^{\circ} \mathrm{C}$. For the polyester we expect a high $\mathrm{T}_{\mathrm{g}}$ value which has a $\mathrm{T}_{\mathrm{g}}$ values higher than $120^{\circ} \mathrm{C}$. And the $\mathrm{T}_{\mathrm{g}}$ values decreased with decreasing rigidity and symmetry of the polymer backbone, which is due to the increase of monomer 1 content.

The thermal stabilities of the polyesters were evaluated by TGA at $20^{\circ} \mathrm{C} / \mathrm{min}$ up to $600^{\circ} \mathrm{C}$ under nitrogen atmosphere. Fig. 5 shows a typical temperature dependence profile of the polyesters weight loss. The $p$-oxybenzoyl homopolyester presents a maximum rate of the thermal decomposition tempe- 
TABLE-2

DATA RESULTS FOR THE POLYESTER

\begin{tabular}{cllcc} 
CODE & $\mathrm{T}_{0}\left({ }^{\circ} \mathrm{C}\right)^{1}$ & $\mathrm{~T}_{10}\left({ }^{\circ} \mathrm{C}\right)^{2}$ & Char yield $^{3}(\%)$ & $\mathrm{Tg}\left({ }^{\circ} \mathrm{C}\right)^{4}$ \\
\hline POB & 419.2 & 460.7 & 59.35 & 349 \\
PPOB & 228.9 & 293.4 & 55.48 & 215 \\
\hline
\end{tabular}

[nitial decomposition temperature recorded by TGA at a heating rate of $20{ }^{\circ} \mathrm{C} / \mathrm{min}$ in $\mathrm{N}_{2},{ }^{2}$ Temperature at which $10 \%$ weight loss is observed, Anaerobic residual weight at $600^{\circ} \mathrm{C},{ }^{4} \mathrm{Glass}$ transition temperature measured on DSC at a heating rate of $10{ }^{\circ} \mathrm{C} / \mathrm{min}$ in $\mathrm{N}_{2}$

rature at $513{ }^{\circ} \mathrm{C}$, yielding a char residue of 59.35 at $600{ }^{\circ} \mathrm{C}$, while a char residue of $55.48 \%$ at $600{ }^{\circ} \mathrm{C}$ was observed in the thermal decomposition of the poly(phosphazene-aryl ester). The two decomposition steps for poly(phosphazenearyl ester) occur at 228.9 and $293.4{ }^{\circ} \mathrm{C}$, respectively. The poly(phosphazene-aryl ester) loss $8 \%$ of the sample in the temperature range $100-250{ }^{\circ} \mathrm{C}$ and another $35.4 \%$ in the range 250-600 ${ }^{\circ}$ C. $p$-Oxybenzoyl homopolyester loss $30.5 \%$ of the sample in temperature range $500-600{ }^{\circ} \mathrm{C}$. Comparing the curves in Fig. 5, it emerges that the first decomposition step of the diol monomer occurs around $150-350{ }^{\circ} \mathrm{C}$. This is attributed to the less stable of the $\mathrm{P}-\mathrm{O}-\mathrm{C}$ bond linkage, as reported for other polymeric systems containing similar polyphosphazne groups $^{9,10}$. It also determines the lower thermal stability of the fully aromatic poly(phosphazene-aryl ester) with respect to their no-polyphosphazene samples.

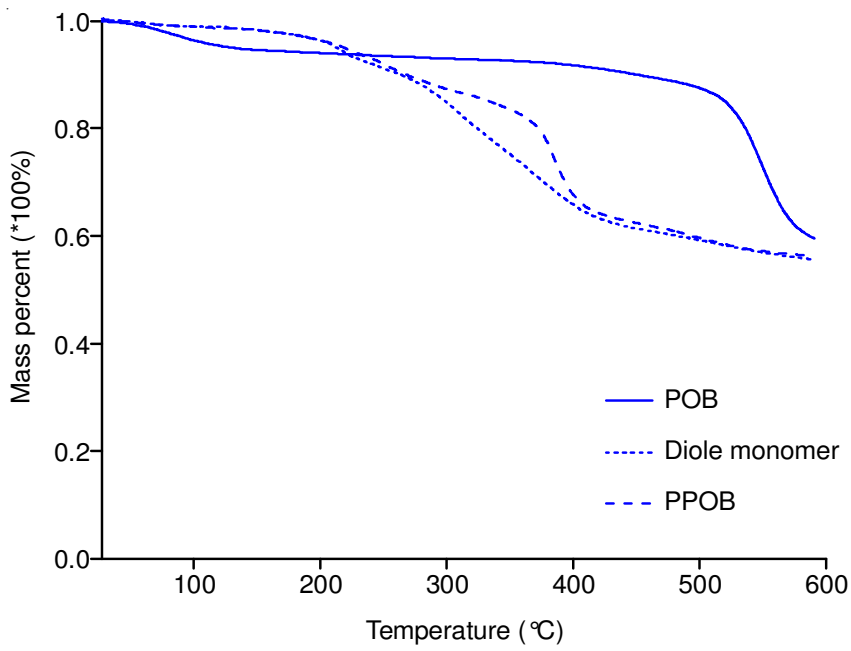

Fig. 5. TGA curves for the polyesters

Thermal degradation properties: Thermogravimetric analysis results indicated that polyphosphazenes play an important roals in increasing the themal stability of poly(phosphazene-aryl ester) at elevated temperatures. However, its stability at lower temperatures decreases. To further investigate their influence, the residues of the polyester samples obtained from the TGA measurements, from room temperature to $600{ }^{\circ} \mathrm{C}$ with a heating rate of $20^{\circ} \mathrm{C} / \mathrm{min}$ in air, were analyzed by FT-IT and SEM. Fig. 6 illustrates the FT-IR spectra of the pyrolysis residues. In the FT-IR spectra of poly(phosphazenearyl ester), the absorption peak at $940 \mathrm{~cm}^{-1}$ due to $\mathrm{P}-\mathrm{O}-\mathrm{C}$ bond and the absorption peak at $1500 \mathrm{~cm}^{-1}$ due to $\mathrm{N}-\mathrm{H}$ bending and $\mathrm{C}-\mathrm{N}$ stretching disappear. The characteristic absorption peaks for $\mathrm{P}=\mathrm{N}$ at about $1230 \mathrm{~cm}^{-1}, \mathrm{P}-\mathrm{N}$ at $875 \mathrm{~cm}^{-1}$ and for ether group at 1180 also disappear. However, a few new peaks appear at $1056 \mathrm{~cm}^{-1}$, which might be ascribed to the generation of P-O-P. Similar to the mechanism which has been reported ${ }^{11,12}$. The obtained structure could act as an acid catalyst, accelerating the cleavage of side groups and the breaking of ether groups in poly(phosphazene-aryl ester). Then, the $p$-oxybenzoyl homopolyester reacts to form more stable structures. The appearance of P-O-P group is considered as crosslinking to different species, resulting in the formation of complex phosphorus structures. This is why the thermal degradation of the poly(phosphazene-aryl ester) is much slower than p-oxybenzoyl homopolyester at above $500{ }^{\circ} \mathrm{C}$.

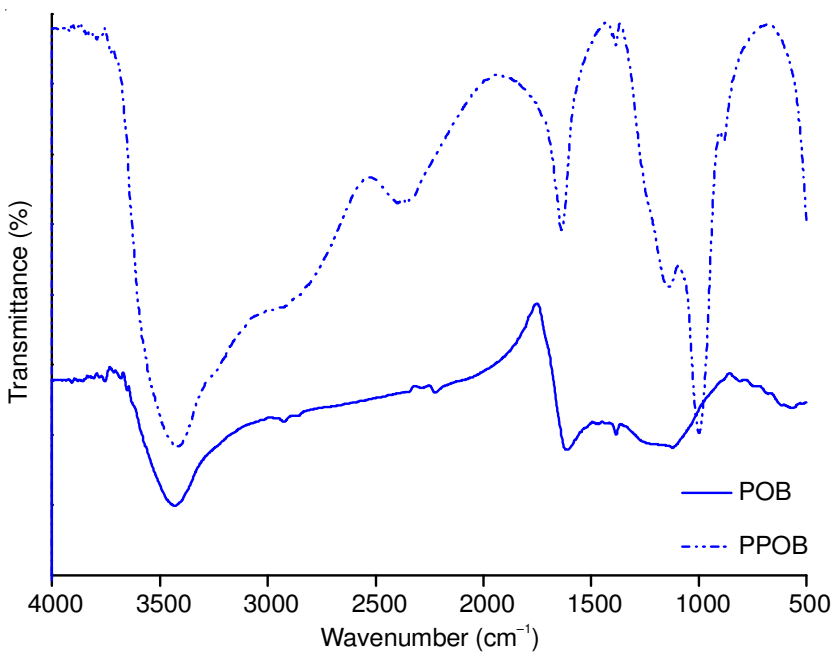

Fig. 6. FT-IR curves for the solid residues of polyesters after pyrolysis

The morphology of the solid residues was observed by scanning electron microscope (Fig. 7). The surface of monomer (Fig. 7c) residues exhibits a porous texture reverse to the lump of $p$-oxybenzoyl homopolyester (Fig. 7a). Comparing with the images (Fig. 7), we can see that the granular of the solid residues gradual disappearance with the increase of monomer content. The surface layer of poly(phosphazene-aryl ester) solid residues has been grumous, for the syneresis of P-O-P took place. It proves that the cyclotriphosphazene moieties produce phosphoric acid or metaphosphoric acid during pyrolysis which acts in the condensed phase promoting char formation on the surface.

\section{Conclusion}

New kinds of poly(phosphazene-aryl ester) was prepared by polycondensation in solution of the our synthesized diols monomer with terephthaloyl dichloride. The diols monomer was characterized by elemental analysis, ${ }^{1} \mathrm{H}$ NMR, ${ }^{31} \mathrm{P}$ NMR and FT-IR techniques to verify its chemical structure. The investigation on thermal properties shows that the thermal stability of our synthesized fully aromatic polyphosphazene polyester is strong determined by the presence of the polyphosphazene groups in the polymer chains. They thermally degradaion in two steps and this behavior may be due to the decomposition of the P-O-C bond and aromatic bonds. The surface of poly(phosphazene-aryl ester) solid residues exhibits a porous texture with the scanning electron microscope. During pyrolysis, polyphosphazene ont only acted in the condensed phase to promote char formation at the surface but also promoted gelation to restrain pyrolysis indirectly. 

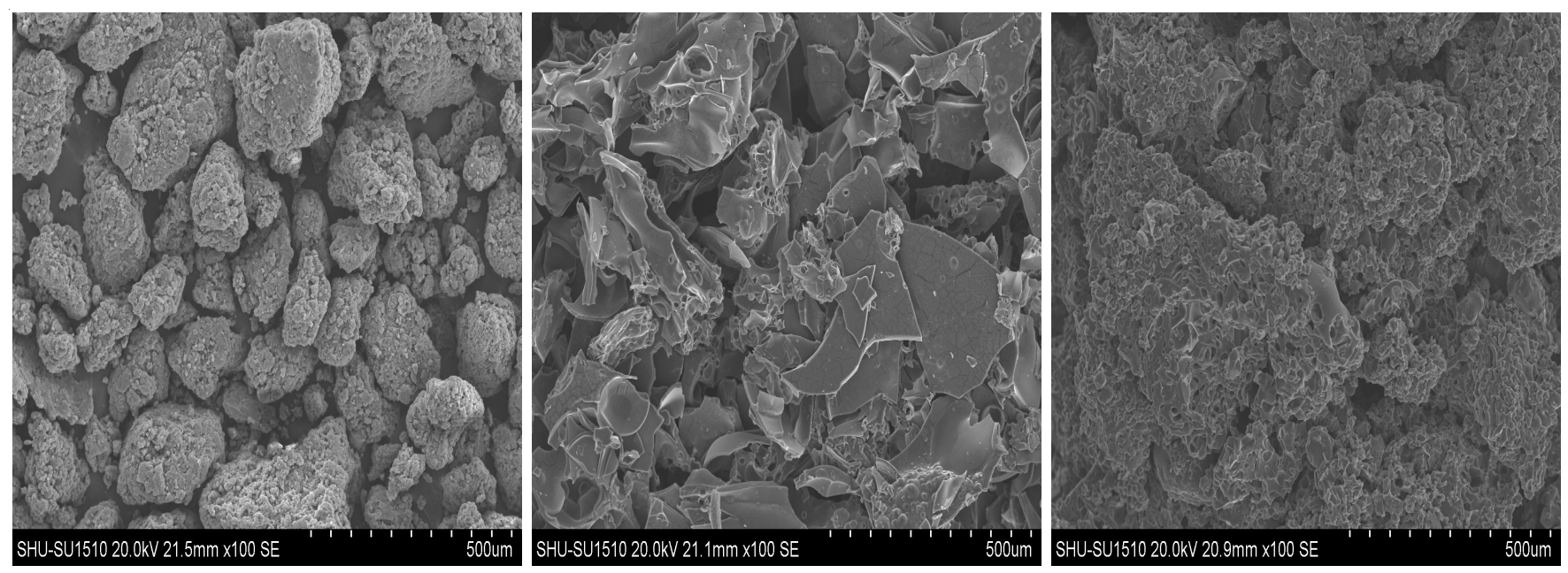

Fig. 7. Morphology of the solid residues of the samples

\section{REFERENCES}

1. K.M. Abraham, M. Alamgir and S.J. Perrotti, J. Electrochem., 135, 535 (1988).

2. H.R. Allock, in ed.: E.J. Glass, Water Soluble Polyphosphazenes and their Hydrogels, In: Hydrophilic Polymers, Adv. Chem. Ser., vol. 248, pp. 3-29 (1996).

3. H.R. Allcock, C.G. Cameron, T.W. Skloss, S. Taylor-Meyers and J.F. Haw, Macromoleules, 29, 233 (1996).

4. H.R. Allcock, C.A. Crane, C.T. Morrissey, J.M. Nelson, S.D. Reeves, C.H. Honeyman and I. Manners, Macromoleules, 29, 7740 (1996).

5. H.R. Allcock and W.E. Krause, Macromoleules, 30, 5683 (1997).
6. H.R. Allcock, W.R. Laredo and R.V. Morford, Solid State Ionics, 139, 27 (2001).

7. H.R. Allcock, J.M. Nelson, R. Prange, C.A. Crane and C.R. de Denus, Macromoleules, 32, 5736 (1999).

8. K. Horie, Y. Morita and Y. Mikamori, M. Suzuli and S. Yano, Patent number: 4382914 (1983).

9. M. El Gouri, A. El Bachiri, S.E. Hegazi, M. Rafik and A. El Harfi, Polym. Degrad. Stab., 94, 2101 (2009).

10. H.-S. Wu and D.-Y. Ke, J. Polym. Res., 5, 95 (1998).

11. R. Liu and X. Wang, Polym. Degrad. Stab., 94, 617 (2009).

12. S.J. Maynard, T.R. Sharp and J.F. Haw, Macromoleules, 24, 2794 (1991). 